Honam Mathematical J. 33 (2011), No. 3, pp. 347-353

http://dx.doi.org/10.5831/HMJ.2011.33.3.347

\title{
REMARKS ON THE MAFFEI'S ISOMORPHISM
}

\author{
NAMHEE KWON
}

\begin{abstract}
In [1], Maffei proved a certain relationship between quiver varieties of type $A$ and the geometry of partial flag varieties over the nilpotent cone. This relation was conjectured by Nakajima, and Nakajima proved his conjecture for a simple case. In the Maffei's proof, the key step was a reduction of the general case of the conjecture to the simple case treated by Nakajima through a certain isomorphism. In this paper, we study properties of this isomorphism.
\end{abstract}

\section{Introduction and Preliminaries}

Quiver varieties of type $A$. Let $I=\{1,2, \cdots, n-1\}$ be the set of vertices of the Dynkin diagram of $\mathfrak{s l}_{n}(\mathbb{C})$. Let $V=\left(V_{i}\right)_{i \in I}$ and $W=\left(W_{i}\right)_{i \in I}$ be collections of finite-dimensional vector spaces. Let us write $\mathbf{v}$ and $\mathbf{w}$ for $\left(\operatorname{dim} V_{1}, \cdots, \operatorname{dim} V_{n-1}\right)$ and $\left(\operatorname{dim} W_{1}, \cdots, \operatorname{dim} W_{n-1}\right)$, respectively. Then we define

$$
\begin{aligned}
M(\mathbf{v}, \mathbf{w})= & \left(\bigoplus_{k=1}^{n-2} \operatorname{Hom}\left(V_{k}, V_{k+1}\right)\right) \oplus\left(\bigoplus_{k=1}^{n-2} \operatorname{Hom}\left(V_{k+1}, V_{k}\right)\right) \\
& \oplus\left(\bigoplus_{k=1}^{n-1} \operatorname{Hom}\left(W_{k}, V_{k}\right)\right) \oplus\left(\bigoplus_{k=1}^{n-1} \operatorname{Hom}\left(V_{k}, W_{k}\right)\right) .
\end{aligned}
$$

Each of four components of an elements of $M(\mathbf{v}, \mathbf{w})$ will be denoted by $A=\left(A_{k}\right), B=\left(B_{k}\right), i=\left(i_{k}\right)$ and $j=\left(j_{k}\right)$.

Define actions of $G_{\mathbf{v}}=\prod_{k=1}^{n-1} G L\left(V_{k}\right)$ and $G_{\mathbf{w}}=\prod G L\left(W_{i}\right)$ on $M(\mathbf{v}, \mathbf{w})$ as follows:

Received June 15, 2011. Accepted August 2, 2011.

2000 Mathematics Subject Classification. 17B37, 16G20.

Key words and phrases. Quiver varieties, group actions, Maffei's isomorphisms.

This research was supported by the Daegu University Research Grant, 2011 
If $g=\left(g_{k}\right) \in G_{\mathbf{v}}$ and $h=\left(h_{i}\right) \in G_{\mathbf{w}}$, then

$g \cdot\left(\left(A_{k}\right),\left(B_{k}\right),\left(i_{k}\right),\left(j_{k}\right)\right)=\left(\left(g_{k+1} A_{k} g_{k}^{-1}\right),\left(g_{k} B_{k} g_{k+1}^{-1}\right),\left(g_{k} i_{k}\right),\left(j_{k} g_{k}^{-1}\right)\right)$

and

$$
h \cdot\left(\left(A_{k}\right),\left(B_{k}\right),\left(i_{k}\right),\left(j_{k}\right)\right)=\left(\left(A_{k}\right),\left(B_{k}\right),\left(i_{k} h_{k}^{-1}\right),\left(h_{k} j_{k}\right)\right) .
$$

Next, we consider a subset $\mu(\mathbf{v}, \mathbf{w})$ of $M(\mathbf{v}, \mathbf{w})$ consisting of quadruples $(A, B, i, j)$ subject to the conditions $B_{1} A_{1}=i_{1} j_{1}, B_{k} A_{k}=A_{k-1} B_{k-1}+$ $i_{k} j_{k}$ for $2 \leq k \leq n-2$, and $A_{n-2} B_{n-2}+i_{n-1} j_{n-1}=0$. An element $(A, B, i, j) \in \mu(\mathbf{v}, \mathbf{w})$ is called stable if each subspace $U=\left(U_{1}, \cdots, U_{n-1}\right)$ of $V=\left(V_{1}, \cdots, V_{n-1}\right)$, which contains $\operatorname{Im}(i)$ (i.e., $\left.\operatorname{Im}\left(i_{k}\right) \subset U_{k}\right)$ and invariant under $A$ and $B$ (i.e., $A_{k}\left(U_{k}\right) \subset U_{k+1}$ and $\left.B_{k}\left(U_{k+1}\right) \subset U_{k}\right)$, is actually equal to $V$. We denote by $\mu(\mathbf{v}, \mathbf{w})^{s}$ the set of stable elements in $\mu(\mathbf{v}, \mathbf{w})$. It is known that the sets $\mu(\mathbf{v}, \mathbf{w})$ and $\mu(\mathbf{v}, \mathbf{w})^{s}$ are invariant under the action of $G_{\mathbf{v}}[3]$.

Maffei's isomorphism. By the framed quiver $Q$ of type $A_{n-1}$ we mean the following diagram:

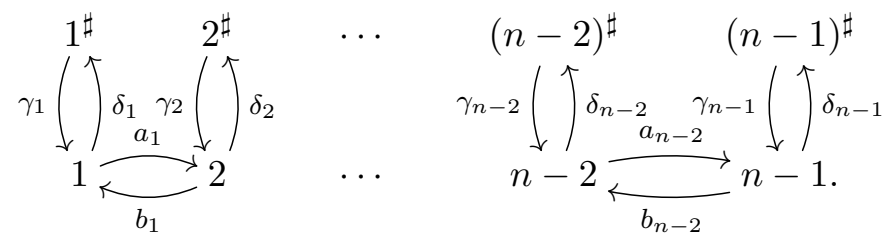

We write

$$
I=\left\{1,2, \cdots, n-1,1^{\sharp}, 2^{\sharp}, \cdots,(n-1)^{\sharp}\right\}
$$

and

$$
H=\left\{a_{1}, \cdots, a_{n-2}, b_{1}, \cdots, b_{n-2}, \gamma_{1}, \cdots, \gamma_{n-1}, \delta_{1}, \cdots, \delta_{n-1}\right\}
$$

for the set of the vertices of $Q$ and the set of arrows of $Q$, respectively.

For a given arrow $h$ of $Q$, we denote the outgoing and incoming vertices of $h$ by out $(h)$ and in $(h)$ respectively. A path $\alpha$ in $Q$ is a sequence $h_{m} h_{m-1} \cdots h_{1}$ of arrows such that in $\left(h_{i}\right)=$ out $\left(h_{i+1}\right)$ for $i=1,2, \cdots, m-1$. We may consider a vertex $i$ as the empty path $\emptyset_{i}$ whose outgoing and incoming vertices are equal to $i$. We call a path $\alpha=h_{m} \cdots h_{1}$ in $Q$ an admissible path if out $\left(h_{1}\right)$, in $\left(h_{m}\right) \in$ $\left\{1^{\sharp}, \cdots,(n-1)^{\sharp}\right\}$. For a given path $\alpha$ in $Q$, we define its evaluation on $M(\mathbf{v}, \mathbf{w})$ as follows: 
If $\alpha$ is an arrow $h$ or an empty path in $Q$, then we define

$$
\alpha(A, B, i, j)= \begin{cases}i d_{V_{k}} & \text { if } \alpha=\emptyset_{k} \text { and } k \in\{1, \cdots, n-1\}, \\ i d_{W_{k}} & \text { if } \alpha=\emptyset_{k^{\sharp}} \text { and } k^{\sharp} \in\left\{1^{\sharp}, \cdots,(n-1)^{\sharp}\right\}, \\ A_{k} & \text { if } \alpha=a_{k}, \\ B_{k} & \text { if } \alpha=b_{k}, \\ i_{k} & \text { if } \alpha=\gamma_{k}, \\ j_{k} & \text { if } \alpha=\delta_{k} .\end{cases}
$$

If $\alpha$ is a path with sequence $\alpha=h_{m} \cdots h_{1}$ of arrows $h_{i}$, then the evaluation of $\alpha$ at $(A, B, i, j)$ is

$$
\alpha(A, B, i, j)=h_{m}(A, B, i, j) \cdots h_{1}(A, B, i, j) .
$$

Let $\mathbf{v}, \mathbf{w}$ be as above. Then, we construct

$$
\tilde{v}_{k}=v_{k}+\sum_{l=k+1}^{n-1}(l-k) w_{l} \text { for } k=1,2, \cdots, n-1,
$$

and

$$
\tilde{w}_{1}=\sum_{k=1}^{n-1} k w_{k} \text { and } \tilde{w}_{k}=0 \text { for } k \geq 2 .
$$

The corresponding vector spaces $\tilde{V}_{k}$ and $\tilde{W}_{k}$ are

$$
\tilde{V}_{k}=V_{k} \oplus\left(\bigoplus_{1 \leq m \leq l-k \leq n-k-1} W_{l}^{(m)}\right) \text { for } k=1,2, \cdots, n-1
$$

and

$$
\tilde{W}_{1}=\bigoplus_{1 \leq m \leq l \leq n-1} W_{l}^{(m)}, \text { and } \tilde{W}_{k}=0 \text { for } k=2, \cdots, n-1,
$$

where $W_{l}^{(m)}$ is an isomorphic copy of $W_{l}$.

We notice that $\operatorname{dim} \tilde{V}_{k}=\tilde{v}_{k}, \operatorname{dim} \tilde{W}_{k}=\tilde{w}_{k}$. We also note that there is a natural embedding of $\prod_{k=1}^{n-1} G L\left(V_{k}\right)$ into $\prod_{k=1}^{n-1} G L\left(\tilde{V}_{k}\right)$. Thus the group $\prod_{k=1}^{n-1} G L\left(V_{k}\right)$ can act on $\mu(\tilde{\mathbf{v}}, \tilde{\mathbf{w}})$ via the natural embedding $\prod G L\left(V_{k}\right) \hookrightarrow \prod G L\left(\tilde{V}_{k}\right)$.

In [1], Maffei constructed $G_{\mathbf{v}}$-equivariant isomorphism $\Phi$ from $\mu(\mathbf{v}, \mathbf{w})$ to a certain transversal subvariety $\mathcal{T}$ of $\mu(\tilde{\mathbf{v}}, \tilde{\mathbf{w}})$. (We refer to [1, Section $3.2]$ for the geometric definition of $\mathcal{T}$. Anyway, we will give an explicit combinatorial description of $\mathcal{T}$ below.) Furthermore, he showed that 
$\Phi(x) \in \mu(\tilde{\mathbf{v}}, \tilde{\mathbf{w}})^{S} \cap \mathcal{T}$ if and only if $x \in \mu(\mathbf{v}, \mathbf{w})^{s}$, and $\left.\Phi\right|_{\mu(\mathbf{v}, \mathbf{w})^{s}}$ is still a $G_{\mathbf{v}}$-equivariant isomorphism.

We now give a more detailed description of the map $\Phi: \mu(\mathbf{v}, \mathbf{w}) \longrightarrow$ $\mathcal{T} \subset \mu(\tilde{\mathbf{v}}, \tilde{\mathbf{w}})$. For an element $(A, B, i, j) \in \mu(\mathbf{v}, \mathbf{w})$, let us denote by $(\tilde{A}, \tilde{B}, \tilde{i}, \tilde{j})$ the element $\Phi(A, B, i, j)$. For notational convenience, we will set $\tilde{V}_{0}=\tilde{W}_{1}, \tilde{A}_{0}=\tilde{i}_{1}$ and $\tilde{B}_{0}=\tilde{j}_{1}$. Then we can consider the maps $\tilde{A}_{k}: \tilde{V}_{k} \longrightarrow \tilde{V}_{k+1}$ and $\tilde{B}_{k}: \tilde{V}_{k+1} \longrightarrow \tilde{V}_{k}(0 \leq k \leq n-2)$ as blockmatrices with respect to the decomposition appearing in (1.3) and (1.4). We notice that $\tilde{i}_{k}=\tilde{j}_{k}=0$ for $k \geq 2$ because $\tilde{W}_{k}=0$ for $k \geq 2$. Next, let us use the following notations for blocks of $\tilde{A}_{k}$ and $\tilde{B}_{k}$ for $0 \leq k \leq n-2$ :

$$
\begin{array}{ll}
\left.\pi_{W_{l}^{(m)}} \circ \tilde{A}_{k}\right|_{W_{l^{\prime}}^{\left(m^{\prime}\right)}}=\mathbb{T}_{k, l, m}^{l^{\prime}, m^{\prime}}, & \left.\pi_{W_{l}^{(m)}} \circ \tilde{B}_{k}\right|_{W_{l^{\prime}}^{\left(m^{\prime}\right)}}=\mathbb{S}_{k, l, m}^{l^{\prime}, m^{\prime}}, \\
\left.\pi_{W_{l}^{(m)}} \circ \tilde{A}_{k}\right|_{V_{k}}=\mathbb{T}_{k, l, m}^{V}, & \left.\pi_{W_{l}^{(m)}} \circ \tilde{B}_{k}\right|_{V_{k+1}}=\mathbb{S}_{k, l, m}^{V}, \\
\left.\pi_{V_{k+1}} \circ \tilde{A}_{k}\right|_{W_{l^{\prime}}^{\left(m^{\prime}\right)}}=\mathbb{T}_{k, V}^{l^{\prime}, m^{\prime}}, & \left.\pi_{V_{k}} \circ \tilde{B}_{k}\right|_{W_{l^{\prime}}^{\left(m^{\prime}\right)}}=\mathbb{S}_{k, V}^{l^{\prime}, m^{\prime}}, \\
\left.\pi_{V_{k+1}} \circ \tilde{A}_{k}\right|_{V_{k}}=\mathbb{A}_{k}, & \left.\pi_{V_{k}} \circ \tilde{B}_{k+1}\right|_{V_{k}}=\mathbb{B}_{k},
\end{array}
$$

where $\pi_{X}$ indicates a projection onto $X$, and $\left.f\right|_{X}$ means a restriction of $f$ to $X$.

In [1], the author calculated explicitly the above blocks of $\tilde{A}_{k}$ and $\tilde{B}_{k}$ in terms of paths in $Q$ :

$$
\begin{aligned}
\mathbb{T}_{k, l, m}^{l^{\prime}, m^{\prime}} & =t_{k, l, m}^{l^{\prime}, m^{\prime}}((A, B, i, j)), \\
\mathbb{S}_{k, l, m}^{l^{\prime}, m^{\prime}} & =s_{k, l, m}^{l^{\prime}, m^{\prime}}((A, B, i, j)), \\
\mathbb{T}_{k, l, m}^{V} & =0, \\
\mathbb{S}_{k, l, m}^{V} & = \begin{cases}\left(\delta_{l} a_{l-1} \cdots a_{k+1}\right)((A, B, i, j)) & \text { if } m=l+k, \\
0 & \text { otherwise, }\end{cases} \\
\mathbb{T}_{k, V}^{l^{\prime}, m^{\prime}} & = \begin{cases}\left(b_{k+1} \cdots b_{l^{\prime}-1} \gamma_{l^{\prime}}\right)((A, B, i, j)) & \text { if } m^{\prime}=1 \text { and } l^{\prime} \geq k+1 \\
0 & \text { otherwise, }\end{cases} \\
\mathbb{S}_{k, V}^{l^{\prime}, m^{\prime}} & =0, \\
\mathbb{A}_{k} & =A_{k}, \\
\mathbb{B}_{k} & =B_{k},
\end{aligned}
$$

where $t_{k, l, m}^{l^{\prime}, m^{\prime}}$ and $s_{k, l, m}^{l^{\prime}, m^{\prime}}$ are certain admissible paths with source vertex $\left(l^{\prime}\right)^{\sharp}$ and target vertex $l^{\sharp}$. ( In this paper, it is not necessary to know 
explicitly the sequence of arrows of the admissible paths. We only need the information of the initial and terminal vertices of the admissible paths for our calculation.)

From now on, we fix $d=\sum_{k=1}^{n-1} k w_{k}$ and a collection $W=\left(W_{1}, \cdots, W_{n-1}\right)$ of vector spaces with a dimension vector $\mathbf{w}=\left(w_{1}, \cdots, w_{n-1}\right)$. Let $V=\left(V_{1}, \cdots, V_{n-1}\right)$ be any collection of vector spaces with a dimension vector $\mathbf{v}=\left(v_{1}, \cdots, v_{n-1}\right)$. Let $\tilde{V}=\left(\tilde{V}_{k}\right)$ and $\tilde{W}=\left(\tilde{W}_{k}\right)$ be the collection of vector spaces obtained from (1.3) and (1.4).

Recall that the isomorphism $\Phi$ is $G_{\mathbf{v}}$-equivariant. Anyway, it is not $G_{\mathrm{w}}$-equivariant in general. Thus, it is not obvious whether the isomorphism $\Phi$ preserves $G_{\mathbf{w}}$-fixed points or not. However, in this paper we prove that the isomorphism $\Phi: \mu(\mathbf{v}, \mathbf{w}) \longrightarrow \mathcal{T} \subset \mu(\tilde{\mathbf{v}}, \tilde{\mathbf{w}})$ actually preserves the set of fixed points under the action of $G_{\mathbf{w}}$. This is the main result of this paper. In addition, we also show that the isomorphism $\Phi$ yields an isomorphism between the $G_{\mathbf{v}^{-}}$orbit spaces on the stable $G_{\mathbf{w}^{-}}$ fixed point subvarieties of $\mu(\mathbf{v}, \mathbf{w})$ and $\mathcal{T}$.

\section{Main results}

We first explain how the group $G_{\mathbf{w}}$ acts on the variety $\mu(\tilde{\mathbf{v}}, \tilde{\mathbf{w}})$. By Equation (1.2), the group $G_{\tilde{\mathbf{w}}}$ acts on the variety $\mu(\tilde{\mathbf{v}}, \tilde{\mathbf{w}})$. Moreover, since $d=\sum_{k=1}^{n-1} k w_{k}$, the group $G_{\mathbf{w}}$ can be diagonally embedded in $G L\left(\mathbb{C}^{d}\right)$, and the group $G L\left(\mathbb{C}^{d}\right)$ is isomorphic to $G L\left(\tilde{W}_{1}\right)=G_{\tilde{\mathbf{w}}}$ because $\operatorname{dim} \tilde{W}_{1}=d$ and $\tilde{W}_{k}=0$ for $k \geq 2$. In this way, we obtain a $G_{\mathbf{w}}$-action on $\mu(\tilde{\mathbf{v}}, \tilde{\mathbf{w}})$. For $g \in G_{\mathbf{w}}$, we denote by $\mu(\mathbf{v}, \mathbf{w})^{g}$ (resp., $\left.\mu(\tilde{\mathbf{v}}, \tilde{\mathbf{w}})^{g}\right)$ the $g$-fixed point subvarieties in $\mu(\mathbf{v}, \mathbf{w})$ (resp., $\left.\mu(\tilde{\mathbf{v}}, \tilde{\mathbf{w}})\right)$.

In the following theorem, we show that the isomorphism $\Phi$ preserves the $G_{\mathbf{w}}$-fixed points though it is not $G_{\mathbf{w}}$-equivariant.

Theorem 2.1. Let $g \in G_{\mathbf{w}}$ and let $\mathcal{T}^{g}=\mathcal{T} \cap \mu(\tilde{\mathbf{v}}, \tilde{\mathbf{w}})^{g}$. Then, for $(A, B, i, j) \in \mu(\mathbf{v}, \mathbf{w})^{g}$ we have $\Phi(A, B, i, j) \in \mathcal{T}^{g}$.

Proof. Let $(A, B, i, j) \in \mu(\mathbf{v}, \mathbf{w})^{g}$, and let $(\tilde{A}, \tilde{B}, \tilde{i}, \tilde{j})=\Phi(A, B, i, j)$. Then $g \cdot(\tilde{A}, \tilde{B}, \tilde{i}, \tilde{j})=\left(\tilde{A}, \tilde{B}, \tilde{i} g^{-1}, g \tilde{j}\right)$. We will show that $\tilde{i} g^{-1}=\tilde{i}$ and $g \tilde{j}=\tilde{j}$. 
We first notice that $\tilde{i}=\tilde{i}_{1}=\tilde{A}_{0}$ because $\bigoplus_{k=1}^{n-1} \operatorname{Hom}\left(\tilde{W}_{k}, \tilde{V}_{k}\right)=$ $\operatorname{Hom}\left(\tilde{W}_{1}, \tilde{V}_{1}\right)$. So, $\tilde{i}$ has the following block-matrix form:

$$
\begin{aligned}
\left.\pi_{W_{l}^{(m)}} \circ \tilde{A}_{0}\right|_{W_{l^{\prime}}^{\left(m^{\prime}\right)}} & =t_{0, l, m}^{l^{\prime}, m^{\prime}}(A, B, i, j), \\
\left.\pi_{V_{1}} \circ \tilde{A}_{0}\right|_{W_{l^{\prime}}^{\left(m^{\prime}\right)}} & = \begin{cases}b_{1} \cdots b_{l^{\prime}-1} \gamma_{l^{\prime}}(A, B, i, j) & \text { if } m^{\prime}=1 \text { and } l^{\prime} \geq 1, \\
0 & \text { otherwise. }\end{cases}
\end{aligned}
$$

Recall that $t_{o, l, m}^{l^{\prime}, m^{\prime}}$ is an admissible path with source vertex $\left(l^{\prime}\right)^{\sharp}$ and target vertex $l^{\sharp}$. Thus, we can express $t_{o, l, m}^{l^{\prime}, m^{\prime}}$ as a product $\delta_{l} P \gamma_{l^{\prime}}$ for some path $P$ in the framed quiver $Q$. Hence

$$
\begin{aligned}
t_{o, l, m}^{l^{\prime}, m^{\prime}}(A, B, i, j) & =\delta_{l}(A, B, i, j) P(A, B, i, j) \gamma_{l^{\prime}}(A, B, i, j) \\
& =j_{l} P(A, B, i, j) i_{l^{\prime}},
\end{aligned}
$$

where $P(A, B, i, j)$ indicates an evaluation of the path $P$ at $(A, B, i, j)$.

Also, we have

$$
\begin{aligned}
b_{1} \cdots b_{l^{\prime}-1} \gamma_{l^{\prime}}(A, B, i, j) & =b_{1}(A, B, i, j) \cdots b_{l^{\prime}-1}(A, B, i, j) \gamma_{l^{\prime}}(A, B, i, j) \\
& =B_{1}\left(b_{2} \cdots b_{l^{\prime}-1}(A, B, i, j)\right) i_{l^{\prime}} .
\end{aligned}
$$

On the other hand, $\tilde{i} g^{-1}$ is a block-matrix with blocks

$$
j_{l} P(A, B, i, j) i_{l^{\prime}} g_{l^{\prime}}^{-1} \text { or } B_{1} b_{2} \cdots b_{l^{\prime}-1}(A, B, i, j) i_{l^{\prime}} g_{l^{\prime}}^{-1} .
$$

Since $g \cdot(A, B, i, j)=(A, B, i, j)$, we have $i g^{-1}=i$. Thus, we have

$$
j_{l} P(A, B, i, j) i_{l^{\prime}} g_{l^{\prime}}^{-1}=j_{l} P(A, B, i, j) i_{l^{\prime}}
$$

and

$$
B_{1} b_{2} \cdots b_{l^{\prime}-1}(A, B, i, j) i_{l^{\prime}} g_{l^{\prime}}^{-1}=B_{1} b_{2} \cdots b_{l^{\prime}-1}(A, B, i, j) i_{l^{\prime}} .
$$

These imply that $\tilde{i} g^{-1}=\tilde{i}$. Similarly, we can show that $g \tilde{j}=\tilde{j}$. The theorem now follows.

Corollary 2.2. The isomorphism $\Phi$ induces the restricted isomorphism $\Phi \mid: \mu(\mathbf{v}, \mathbf{w})^{g} \longrightarrow \mathcal{T}^{g}$ for each $g \in G_{\mathbf{w}}$. by

Proof. It is known from [1, Lemma 19] that the inverse of $\Phi$ is given

$$
\Phi^{-1}(\tilde{A}, \tilde{B}, \tilde{i}, \tilde{j})=\left(\left(\mathbb{A}_{k}\right),\left(\mathbb{B}_{k}\right),\left(\mathbb{T}_{k, V}^{k+1,1}\right),\left(\mathbb{S}_{k, k+1,1}^{V}\right)\right) .
$$


Thus, $\Phi^{-1}$ induces a morphism $\Phi^{-1}: \mathcal{T}^{g} \longrightarrow \mu(\mathbf{v}, \mathbf{w})^{g}$ because $\mathbb{A}_{k}$, $\mathbb{B}_{k}, \mathbb{T}_{k, V}^{k+1,1}$ and $\mathbb{S}_{k, k+1,1}^{V}$ are blocks of $\tilde{A}_{k}$ and $\tilde{B}_{k}$. The corollary now follows.

For $g \in G_{\mathbf{w}}$, we denote by $\mu(\mathbf{v}, \mathbf{w})^{g, s}$ the set of stable elements in $\mu(\mathbf{v}, \mathbf{w})^{g}$. Similarly, we write $\mathcal{T}^{g, s}$ for the set of stable elements in $\mathcal{T}^{g}$. Recall that the $G_{\mathbf{w}}$-action commutes with the $G_{\mathbf{v}}$-action [3]. Thus, the induced action of $G_{\mathbf{w}}$ on $\mu(\tilde{\mathbf{v}}, \tilde{\mathbf{w}})$ also commutes with the $G_{\tilde{\mathbf{v}}}$-action because $G_{\mathbf{w}}$ acts on $\mu(\tilde{\mathbf{v}}, \tilde{\mathbf{w}})$ via the embedding $G_{\mathbf{w}} \hookrightarrow G_{\tilde{\mathbf{w}}}$. Hence, we obtain the orbit spaces $\mu(\mathbf{v}, \mathbf{w})^{g, s t a b} / G_{\mathbf{v}}$ and $\mathcal{T}^{g, s t a b} / G_{\tilde{\mathbf{v}}}$.

As a by-product of Theorem 2.1, we have the following corollary.

Corollary 2.3. The isomorphism $\Phi$ yields an isomorphism

$$
\tilde{\varphi}: \mu(\mathbf{v}, \mathbf{w})^{g, s} / G_{\mathbf{v}} \longrightarrow \mathcal{T}^{g, s} / G_{\tilde{\mathbf{v}}}
$$

defined by $\tilde{\varphi}\left(G_{\mathbf{v}}(A, B, i, j)\right)=p(\Phi((A, B, i, j)))$, where $g \in G_{\mathbf{w}},(A, B, i$, $j) \in \mu(\mathbf{v}, \mathbf{w})^{g, s}$ and $p$ is the natural projection $p: \mu(\tilde{\mathbf{v}}, \tilde{\mathbf{w}})^{s} \longrightarrow \mu(\tilde{\mathbf{v}}, \tilde{\mathbf{w}})^{s} / G_{\tilde{\mathbf{v}}}$.

Proof. We recall that the $G_{\mathbf{v}^{-}}$equivariant isomorphism $\Phi$ preserves the stability, and its restriction $\left.\Phi\right|_{\mu(\mathbf{v}, \mathbf{w})^{s}}: \mu(\mathbf{v}, \mathbf{w})^{s} \longrightarrow \mathcal{T}^{s}$ is an isomorphism [1]. Here $\mathcal{T}^{s}$ indicates the set $\mu(\tilde{\mathbf{v}}, \tilde{\mathbf{w}})^{s} \cap \mathcal{T}$. Hence, by Theorem (2.1) the map $\tilde{\varphi}$ is well-defined and surjective.

In addition, according to [1, Definition 20] and [1, Theorem 8] the morphism

$$
\psi: \mu(\mathbf{v}, \mathbf{w})^{s} / G_{\mathbf{v}} \longrightarrow \mathcal{T}^{s} / G_{\tilde{\mathbf{v}}}
$$

defined by $\psi\left(G_{\mathbf{v}}(A, B, i, j)\right)=p(\Phi((A, B, i, j)))$ is an isomorphism.

Notice that $\tilde{\varphi}$ is the restriction of $\psi$ to the subvariety $\mu(\mathbf{v}, \mathbf{w})^{g, s} / G_{\mathbf{v}}$. Thus, $\tilde{\varphi}$ is injective. The corollary now follows.

\title{
References
}

[1] A. Maffei, Quiver varieties of type A, Comment. Math. Helv. 80 (2005), 1-27.

[2] H. Nakajima, Instantons on ALE spaces, quiver varieties and Kac-Moody algebras, Duke Math. J. 76 (1994), 365-416.

[3] H. Nakajima, Quiver varieties and finite dimensional representations of quantum affine algebras, J. Amer. Math. Soc. 14 (2001), 145-238.

\author{
Namhee Kwon \\ Department of Mathematics, Daegu University, \\ Daegu, 712-714, Korea. \\ E-mail: nkwon@daegu.ac.kr
}

